eCommons@AKU

July 2011

\title{
Intracranial atherosclerotic disease
}

\author{
Maria Khan \\ Aga Khan University \\ Imama Naqvi \\ Aga Khan University \\ Asha Bansari \\ Aga Khan University \\ Ayeesha Kamran Kamal \\ Aga Khan University
}

Follow this and additional works at: https://ecommons.aku.edu/pakistan_fhs_mc_med_med Part of the Cardiovascular Diseases Commons

\section{Recommended Citation}

Khan, M., Naqvi, I., Bansari, A., Kamal, A. (2011). Intracranial atherosclerotic disease. Stroke Research and Treatment., 2011. Available at: https://ecommons.aku.edu/pakistan_fhs_mc_med_med/62 


\title{
Intracranial Atherosclerotic Disease
}

\author{
Maria Khan, ${ }^{1}$ Imama Naqvi, ${ }^{2}$ Asha Bansari, ${ }^{2}$ and Ayeesha Kamran Kamal ${ }^{3}$ \\ ${ }^{1}$ Fellow International Cerebrovascular Translational Clinical Research Training Program, Stroke Service, \\ Aga Khan University Hospital, Karachi 74800, Pakistan \\ ${ }^{2}$ Research Officers, Medical College, Aga Khan University Hospital, Karachi 74800, Pakistan \\ ${ }^{3}$ Director Stroke Service and Vascular Fellowship Program, Section of Neurology, Department of Medicine, \\ Aga Khan University Hospital, Karachi 74800, Pakistan
}

Correspondence should be addressed to Ayeesha Kamran Kamal, kamalsnyc@aol.com

Received 9 December 2010; Revised 13 April 2011; Accepted 2 May 2011

Academic Editor: Scott E. Kasner

Copyright (C) 2011 Maria Khan et al. This is an open access article distributed under the Creative Commons Attribution License, which permits unrestricted use, distribution, and reproduction in any medium, provided the original work is properly cited.

\begin{abstract}
Intracranial atherosclerotic disease (ICAD) is the most common proximate mechanism of ischemic stroke worldwide. Approximately half of those affected are Asians. For diagnosis of ICAD, intra-arterial angiography is the gold standard to identify extent of stenosis. However, noninvasive techniques including transcranial ultrasound and MRA are now emerging as reliable modalities to exclude moderate to severe (50\%-99\%) stenosis. Little is known about measures for primary prevention of the disease. In terms of secondary prevention of stroke due to intracranial atherosclerotic stenosis, aspirin continues to be the preferred antiplatelet agent although clopidogrel along with aspirin has shown promise in the acute phase. Among Asians, cilostazol has shown a favorable effect on symptomatic stenosis and is of benefit in terms of fewer bleeds. Moreover, aggressive risk factor management alone and in combination with dual antiplatelets been shown to be most effective in this group of patients. Interventional trials on intracranial atherosclerotic stenosis have so far only been carried out among Caucasians and have not yielded consistent results. Since the Asian population is known to be preferentially effected, focused trials need to be performed to establish treatment modalities that are most effective in this population.
\end{abstract}

\section{Introduction}

1.1. Epidemiology. Intracranial atherosclerotic stenosis of the major arteries (intracranial internal carotid artery, middle cerebral artery, vertebral artery, and basilar artery) is the most common proximate mechanism of ischemic stroke worldwide [1]. It causes $30 \%$ to $50 \%$ of strokes in Asians [2] and $8 \%$ to $10 \%$ of strokes in North American Caucasians [3].

Intracranial atherosclerotic disease, ICAD, defined as atherosclerosis of the large arteries at the base of the brain, preferentially affects Asians, Hispanics, Far East Asians, and Blacks as compared to carotid bifurcation disease [3-6]. Also, about $20 \%-45 \%$ of non-Caucasians with large artery disease have combined extracranial and intracranial lesions [7-10]. The prevalence of atherosclerotic stenosis by subtype and race is further reported in Table 1.

\section{Predisposing Factors for ICAD in Susceptible Populations}

2.1. Racial Associations. Sacco et al. [3] found no difference between races in the proportion of patients with extracranial atherosclerotic stroke, while intracranial atherosclerosis was seen more frequently in African American and Hispanic subjects than in Caucasian subjects. As per this study the greater prevalence of Diabetes Mellitus in African American and Hispanic subjects accounted for the increased frequency of ICAD.

Waddy et al. [17] report racial differences between blacks and Caucasians with respect to intracranial stenosis. In this study, risk of recurrence of stroke was higher in blacks and risk factor profiles were also different.

Among Chinese populations, ICAD-related strokes account for $33 \%-37 \%$ of all ischemic strokes and an even higher prevalence is reported from Korea, Thailand, and Singapore $[15,16]$. Hence, there is evidence of racial association with incidence of ICAD.

2.2. Genetic Associations. Several studies suggest a positive correlation between race and cerebrovascular disease [18, 19]. A study conducted on subjects of European ancestry uncovered a genetic trait that increased their resistance to atherosclerosis, though protection was confined to large 
TABLE 1: Prevalence of intracranial atherosclerotic disease/extracranial atherosclerotic disease by race.

\begin{tabular}{lcc}
\hline Race & ICAD & ECAD \\
\hline South Asians [11, 12] & $20 \%-54 \%$ & $10 \%$ \\
East Asians [11] & $7 \%$ & $18 \%$ \\
US Whites [3, 13] & $1 \%-24 \%$ & $11 \%-33 \%$ \\
US Blacks [3, 13] & $6 \%-22 \%$ & $8 \%-15 \%$ \\
US Hispanics [3] & $11 \%$ & $9 \%$ \\
Chinese [14] & $33 \%-50 \%$ & $19 \%$ \\
Koreans [1, 15] & $56.3 \%{ }^{\dagger}-26.4 \%$ & $12.2 \%$ \\
Singapore [1] & $47.9 \%$ & $\mathrm{NR}$ \\
Thailand [16] & $47 \%$ & $49 \%$ \\
\hline
\end{tabular}

${ }^{\dagger}$ The authors used $30 \%$ stenosis as cutoff.

ICAD: intracranial atherosclerotic disease, ECAD: extracranial atherosclerotic disease.

intracranial vessels. It was speculated that stroke suppressor genes activated antioxidants, such as Peroxidase 1, a component of high density lipoprotein, which acted predominantly in the intracranial arteries. Hence, polymorphic expression of stroke suppressor genes in different races accounts for variable susceptibility to intracranial disease [20]. Secondary prevention trials have so far failed to demonstrate benefit of antioxidants in reducing atherosclerotic complications [21, $22]$. However, since early atherosclerotic changes begin in the cerebral arteries soon after teenage [23], future antioxidant trials should intervene at very young age to determine a role of antioxidants against the atherosclerotic process.

Most human genetic studies focus on polymorphic variants that promote stroke predisposing phenotypes or mediators like targets in inflammatory pathways, modulators of vascular tone and endothelial functions, lipid and homocysteine metabolisms, and components of haemostatic system. Allelic association studies have so far yielded either conflicting or only weak and modest connections [24-26].

Considering the racial differences in prevalence of ICAD, and its predominance among the Asian population, there is a need for genetic studies to be carried out in different population subsets to further explore the genetic trends. Furthermore, research should explore our innate protective mechanisms against atherosclerosis and their expressions in intracranial and systemic arteries among different races.

2.3. Risk Factor Associations. The reasons for the disposition towards more intracranial occlusive lesions in the Asian population remains unclear although various studies from the past two decades have shown that coronary heart disease, stroke, hypertension, and diabetes mellitus are associated with more extensive cerebral atherosclerosis [4, 27]. Risk factors associated with intracranial atherosclerosis are certain races (Hispanic Americans, blacks, and Asians), female sex, young age, hypertension, smoking, diabetes, and lipid disorders [3, 4, 6, 28, 29].

A WASID (warfarin versus aspirin in symptomatic intracranial disease) substudy [17] has compared risk factors with respect to racial differences amongst blacks and Caucasians. They found that blacks with intracranial stenosis were more likely to have hypertension, diabetes, high LDL, and high total cholesterol. Other studies report that compared to extracranial atherosclerosis, intracranial atherosclerosis is not associated with typical risk factors for peripheral and coronary atherosclerosis: male sex and hypercholesterolemia $[3,4]$.

Metabolic Syndrome, but not its isolated components, has been found to be independently associated with intracranial atherosclerosis, suggesting screening and treatment of metabolic abnormalities as an important prevention strategy [30]. The Northern Manhattan stroke study [31] also showed that the metabolic syndrome confers a greater risk of development of intracranial atherosclerotic strokes compared to extracranial atherothrombotic and non-atherothrombotic strokes.

Another recent analysis [32] on WASID patients has shown that severity of stenosis depends most strongly on lipid disorder but also has an association with diabetes and the metabolic syndrome. The study also highlights that the location of intracranial stenosis depends on different vascular risk factors suggesting that the underlying pathophysiology among different intracranial vessels may be different.

2.4. Clinical and Radiological Presentation of Stroke. Patients with intracranial atherosclerotic disease present with ischemic strokes in the territory of the affected artery. The mechanisms proposed in ischemia include artery-to-artery embolism, local branch occlusion, hemodynamic compromise resulting from progressive arterial narrowing, or a combination of these factors [33].

The radiological presentation of these strokes has been described in several studies. These presentations include cortically based infarctions [34], border zone infarctions [35, 36] and lacunes [37-39]. Multiple cortical and subcortical infarcts have been reported in a study of patients with MCA stenosis [33], and this has been linked to presence of embolic signals on transcranial Doppler (TCD) exam of these patients. This finding suggests artery-to-artery embolism as the likely mechanism for this stroke subtype. Infarctions in the territory of MCA perforators have also been described in another magnetic resonance imaging(MRI-) based study [40] and the underlying mechanism proposed is atherosclerotic branch occlusion.

Both internal and cortical border zone infarctions of the MCA territory are reported in literature to result from ICAD $[41,42]$. With progressive narrowing of the large artery, there is a reduction in flow, and since this happens gradually, leptomeningeal collaterals form. When the compromise reaches a stage when these collaterals are insufficient to maintain perfusion to the terminal areas, infarctions develop in these borderzone territories [43]. Multiple lesions in the unilateral anterior circulation and small scattered lesions in one vascular territory are also related to large-artery atherosclerosis $[33,44,45]$.

2.5. Natural Course and Clinical Prognosis. The natural history of stenosis secondary to intracranial atherosclerosis has been angiographically studied by Akins et al. [46]. Over a follow-up period of 26.7 months, they reported an increase 
in the degree of stenosis in the MCA-ACA-PCA group, whereas intracranial ICA stenoses remained stable. They also reported a $14 \%$ regression in intracranial ICA stenosis and a $28 \%$ regression in the MCA-ACA-PCA group. They concluded that intracranial lesions are dynamic and may progress or regress with time.

A study by Kwon et al. [47], evaluating the role of cilostazol in ICAD, describes both progression and regression in these lesions. In a more recent study [48] evaluating the role of cilostazol and clopidogrel in intracranial atherosclerosis, a total of $14 \%$ patients demonstrated lesion progression.

ICAD has also been evaluated in terms of development of stroke in the territory of the diseased vessel. The risk of future ischemic strokes depends on whether the vessel is asymptomatic or symptomatic. This difference has been well studied in the WASID trial [49]. Patients recruited in the trial, though symptomatic, also had asymptomatic stenoses in other arteries. When these patients were followed up, the 1 -year risk of developing a stroke in the territory of these asymptomatic vessels was 3.5\% (CI $0.8 \%-9 \%$ ). In contrast to this, patients with $\geq 70 \%$ stenosis had a risk of $14 \%$ at one year for a stroke in the same territory and $19 \%$ for stroke in any vascular territory. Apart from severity of stenosis, recent symptoms and female gender also predicted risk of stroke recurrence in this study [50]. A similar risk rate of $14 \%$ was reported from the GESICA (Groupe d'Etude des Stenoses Intra-Crâniennes Athéromateuses symptomatiques) study [51].

A WASID substudy [52] has identified that the presence of collaterals is a strong predictor of subsequent stroke in case of moderate-to-severe stenosis, but milder stenoses are more unstable and presence of collaterals in these predicts an increased risk of subsequent stroke.

2.6. Diagnosis. In patients with clinical suspicion of intracranial steno-occlusive lesions, accurate assessment of intracranial arteries is essential for optimal therapeutic decisions. The options for imaging patients with intracranial stenosis include noninvasive techniques such as transcranial Doppler or magnetic resonance angiography (MRA), such as contrast-enhanced (CE) MRA and CE computerized tomography CT.

Catheter cerebral angiography, though invasive, is considered to be the gold standard for diagnosis of ICAD. The complication rates associated with the procedure performed by trained neurointerventionists have gone down significantly. A recent review [53] of six-year data based on 363 diagnostic angiographies reports a low complication rate of $0.3 \%$ and an even lower risk of stroke $(0.03 \%)$.

MRA is being proposed as a replacement for the gold standard, intra-arterial angiography. 3D time-of-flight (TOF) MRA is currently the most commonly used pulse sequence in the MR evaluation of intracranial arteries. It detects clinically significant stenotic lesions in intracranial vessels. The other potential field of application is to monitor the response of a stenotic lesion to antistenosis medications [47].

Both TOF MRA and CE MRA have shown [54] high accuracy for the detection of high-grade ICA stenosis and occlusions. CE MRA has some edge over TOF MRA. However, for moderately severe stenosis, both had only poor (TOF-MRA) to fair (CE MRA) sensitivity. Also, 3D TOFMRA can be restricted by factors such as susceptibility artifacts near the sphenoid sinus, limited scan range, limited spatial resolution, and flow signal intensity loss due to saturation or phase dispersion $[55,56]$. The portion of intracranial vessels near the skull base and especially the paracavernous and supraclinoid segments of the internal carotid arteries are areas of frequent over and underestimation of stenosis due to the presence of dephasing artifacts. Some of these limitations can be overcome by use of CE MRA which is not flow dependant.

When 3 Tesla MRI is used, the sensitivity of TOF-MRA for $>50 \%$ stenosis is reported to be $78 \%-85 \%$ with a positive predictive value $75 \%-79 \%$, and for complete occlusions, the sensitivity and positive predictive values are in the range of $100 \%$ and $87 \%$, respectively [57].

A recent paper by Arenillas [58] has highlighted a new concept of intracranial plaque imaging using high resolution (3T) 3D TOF-MRA. This gives the advantage of characterization of plaque, detection of nonstenotic intracranial atheroma, and detection of intraplaque hemorrhage. This is yet to come into widespread clinical practice due to nonavailability and limited clinical value. However, it is interesting, because it characterizes lesser degrees of stenosis.

In a comparative study [59], the ability of helical CT angiography (CTA) to help detect and quantify intracranial stenosis and occlusion compared with DSA (digital subtraction angiography) and MRA was evaluated. CTA revealed higher sensitivity than MRA for intracranial stenosis $(98 \%$ versus $70 \%, P<.001)$ and occlusion $(100 \%$ versus $87 \%$, $P=.02)$.

Transcranial Doppler, TCD is another noninvasive and easy to perform modality used for evaluation of ICAD. The stroke outcomes and neuroimaging of intracranial atherosclerosis (SONIA) trial [60] showed that both TCD and MRA identify $50 \%$ to $99 \%$ intracranial large vessel stenosis with a significant negative predictive value. Therefore, both can reliably exclude the presence of intracranial stenosis though abnormal findings would require a confirmatory test such as angiography to reliably identify stenosis.

Yet, another study [61] shows that advanced ultrasonographic techniques like power-flow imaging and color Doppler-assisted duplex imaging with and without contrast have a better yield for near-occlusion and complete occlusion detection.

Available noninvasive imaging modalities and their sensitivity and specificity of detection with respect to degree of stenosis is enlisted in Table 2. Overall, noninvasive imaging modalities have a high negative predictive value in detecting intracranial atherosclerosis. The gold standard for confirmation of the diagnosis remains intra-arterial angiography.

\section{Treatment of Atherosclerotic Intracranial Stenosis}

3.1. Medical Management. The medical management of intracranial atherosclerotic disease has been evaluated in several 
TABLE 2: Comparison of nonimaging modalities in the detection of intracranial stenosis.

\begin{tabular}{|c|c|c|c|c|}
\hline Modality & Degree of stenosis & Sensitivity & Specificity & Limitations \\
\hline $\begin{array}{l}\text { Digital subtraction } \\
\text { angiography }\end{array}$ & & & & $\begin{array}{l}\text { Invasive test: Procedure risk rate: } 0.3 \% \text { for all } \\
\text { complications, } 0.03 \% \text { for stroke }[56]\end{array}$ \\
\hline \multirow{3}{*}{$\begin{array}{l}\text { MRA (TOF) }[54] \text { for } \\
\text { ICA disease }\end{array}$} & $50 \%-69 \%$ & $37.9 \%$ & $92.1 \%$ & \multirow{5}{*}{$\begin{array}{l}\text { Limited spatial resolution, flow signal intensity loss as a } \\
\text { result of saturation or phase dispersion, susceptibility } \\
\text { artifacts near sphenoid sinus, and over- and } \\
\text { underestimation of stenosis due to dephasing artifacts }\end{array}$} \\
\hline & $>70 \%-99 \%$ & $91.2 \%$ & $88.3 \%$ & \\
\hline & ICA occlusion & $94.5 \%$ & $99.3 \%$ & \\
\hline \multirow{2}{*}{ MRA (TOF) 3T [57] } & $50 \%-99 \%$ stenosis & $78 \%-85 \%$ & $95 \%$ & \\
\hline & Occlusion & $100 \%$ & $99 \%$ & \\
\hline \multirow{3}{*}{ MRA (CE) [54] } & $50 \%-69 \%$ & $65.9 \%$ & $93.5 \%$ & \\
\hline & $>70 \%-99 \%$ & $94.6 \%$ & $91.9 \%$ & \\
\hline & ICA occlusion & $99.4 \%$ & $99.6 \%$ & \\
\hline \multirow{2}{*}{$\mathrm{CTA}^{*}[59]$} & Stenosis ${ }^{\ddagger}$ & $98 \%$ & $99 \%$ & \\
\hline & Occlusion & $100 \%$ & $100 \%$ & \\
\hline \multirow[t]{3}{*}{$\begin{array}{l}\text { Transcranial Doppler } \\
\text { Ultrasound [62] }\end{array}$} & $\begin{array}{l}>50 \% \text { stenosis or } \\
\text { occlusion }\end{array}$ & & & \\
\hline & For MCA stem (M1) & $90 \%-99 \%$ & $90 \%-99 \%$ & High level of technical and procedural skill is required \\
\hline & $\begin{array}{c}\text { For intracranial } \\
\text { segment (V4) of } \\
\text { vertebral and basilar } \\
\text { artery }\end{array}$ & $70 \%-80 \%$ & $90 \%-99 \%$ & $\begin{array}{l}\text { to obtain the best quality images. Reliable insonation of } \\
\text { the posterior circulation is particularly difficult }\end{array}$ \\
\hline \multicolumn{5}{|l|}{ CDDI $[61]$} \\
\hline Unenhanced $^{\dagger}$ & \multirow{4}{*}{$\begin{array}{l}\text { Atheromatous } \\
\text { pseudo-occlusion }\end{array}$} & $70 \%$ & $92 \%$ & False negative rate $30 \%$ \\
\hline Echo-enhanced PFI & & $83 \%$ & $92 \%$ & False negative rate $17 \%$ \\
\hline Unenhanced & & $95 \%$ & $92 \%$ & False negative rate $5 \%$ \\
\hline Echo-enhanced & & $94 \%$ & $100 \%$ & False negative rate $6 \%$ \\
\hline
\end{tabular}

CDDI: Color Doppler-assisted duplex imaging, PFI: power-flow imaging.

* Data are percentages using DSA as the reference standard.

${ }^{\ddagger}$ North American Symptomatic Carotid Endarterectomy Trial (NASCET) criteria were used for stenosis calculations: $\left[\left(D_{n}-D_{s}\right) / D_{n}\right] \times 100$, where $D_{n}$ is normal diameter and $D_{s}$ is stenosed diameter. NASCET stenoses were grouped according to the following grading scale: normal (0\%-9\%), mild (10\%-29\%), moderate $(30 \%-69 \%)$, severe $(70 \%-99 \%)$, or occluded (no flow detected). Normal $(0 \%-9 \%)$ and mild $(10 \%-29 \%)$ stenosis were not considered diseased vessel segments and were excluded from analysis.

${ }^{\dagger}$ Ultrasound emission energy and gain cannot be increased high enough without the appearance of disturbing acoustic noise that diminishes the reliable depiction of orthograde flow signals.

trials over the past decade. There is no data on primary prevention of strokes in patients with asymptomatic stenosis.

The effectiveness of Aspirin in secondary prevention of ischemic strokes is irrefutable. Since international stroke trial-IST and chinese acute stroke trial-CAST [63, 64], it is the standard of care. For intracranial atherosclerotic strokes, it has never been tested in isolation, but extrapolating from this data, the benefit spans across all stroke subtypes.

For secondary prevention, anticoagulation with warfarin was shown to be less safe and equal in efficacy to aspirin in the WASID (warfarin versus aspirin in symptomatic intracranial disease) trial [49]. WASID was stopped early after a mean followup of 1.8 years because of higher rates of death and major hemorrhage in the warfarin group. The rates of myocardial infarction or sudden death were also higher in the warfarin group. The primary end point of ischemic stroke, brain hemorrhage or vascular death, occurred in $22.1 \%$ of patients in aspirin and $21.8 \%$ of those in the warfarin group. Before WASID, it was thought that patients with vertebrobasilar disease might benefit from warfarin, but the study failed to show a significantly lower rate of primary end point or stroke in the group on warfarin [65], suggesting that there is no clear evidence for supremacy of warfarin over aspirin for patients with vertebrobasilar stenosis either.

Efficacy of other antiplatelet agents has been evaluated in several other trials, and aspirin/extended release dipyridamole [66] is recommended over aspirin for secondary prevention of all ischemic strokes. Clopidogrel [67] was also shown to be superior to aspirin for composite vascular end points. Therefore even though not subtype specific, there is a role for these agents in ischemic strokes. Another more recent study (prevention regimen for effectively avoiding second strokes-PRoFESS) has shown similar stroke recurrence rates in patients with various underlying causes of stroke and in a subset of patients with large artery atherosclerosis when treated with clopidogrel alone versus a combination of aspirin/extended release dipyridamole [68].

Therefore, as per the American Stroke Association recommendation [69], aspirin alone, aspirin/extended release dipyridamole, and clopidogrel alone are all acceptable 
options for secondary stroke prevention after a non-cardioembolic ischemic stroke including large artery atherosclerotic stroke.

A recent trial [70] investigating the role of combination antiplatelet (aspirin+clopidogrel) in acute management of stroke secondary to large artery atherosclerosis has shown promise. The study demonstrated that early combination therapy (within 7 days of symptom onset) was more effective than aspirin alone in reducing microembolic signals in symptomatic arteries of these patients. Whether this translates into clinical benefit is yet to be evaluated.

Cilostazol is a newer antiplatelet agent being investigated for intracranial stenosis. It is a phosphodiesterase 3 inhibitor that inhibits smooth muscle cell growth in vitro and has an antiatherogenic and antiproliferative action in addition to antiplatelet effects. The first study [47] evaluating cilostazol in a randomized fashion came out in 2005. In this study, during a 6-month follow-up period, there were no strokes in cilostazol+aspirin or placebo+aspirin arm. However, progression of the intracranial stenosis was significantly less in the cilostazol group $(6.7 \%$ versus $28.8 \% ; P=.008)$.

This finding led to a multicenter study of cilostazol+aspirin versus clopidogrel+aspirin in patients with symptomatic intracranial stenosis, the trial of cilostazol in symptomatic intracranial arterial stenosis II (TOSS II) [48]. TOSS II enrolled 456 acute ischemic stroke patients with symptomatic intracranial arterial stenosis. After 7 months of treatment, follow-up MRA showed a slightly lower but significant rate of progression $(9.90 \%$ versus $15.46 \%)$ and a higher rate of regression $(30.20 \%$ versus $23.67 \%)$ in symptomatic stenosis with cilostazol versus clopidogrel group, respectively. More patients in the cilostazol group had new asymptomatic ischemic lesions at the follow-up MRA than those receiving clopidogrel (18.68\% versus $12.04 \%)$, and in the territory of the symptomatic intracranial stenosis $(12.09 \%$ versus $8.90 \%)$, but this difference was not statistically significant. There was no statistically significant difference in the occurrence of clinical events by treatment group although events tended to be more frequent in the cilostazol. Bleeding complications were nonsignificantly higher with clopidogrel. Therefore, cilostazol combination therapy had a favorable effect on the overall change in symptomatic intracranial atherosclerotic stenosis, but in this study, it did not translate into better clinical outcomes.

A pilot study [71] of Chinese patients with ischemic strokes has compared aspirin to cilostazol in a randomized fashion. There were fewer ischemic and hemorrhagic strokes in the cilostazol group, suggesting that it might be safer and more effective compared to aspirin. Cilostazol stroke prevention study 2-CSPS-2 [72] is a more recent randomized trial that has shown that cilostazol is noninferior to and may be superior to aspirin in prevention of recurrent stroke in patients with noncardioembolic ischemic strokes. One third of the patients in this trial had large vessel atherosclerosis.

Management of Risk Factors. WASID presented additional data supporting aggressive risk-factor control in patients with intracranial stenosis. In WASID, vascular risk factors were managed by following national guidelines [48]. However, in many patients risk-factor control was not optimal, and patients with poor control of risk factors had higher rates of recurrent vascular events $[73,74]$.

A WASID-substudy [74] demonstrated that elevated blood pressure was associated with an increased risk of ischemic stroke and other major vascular events. Contrary to the common practice of permissive hypertension in highgrade stenosis, this study showed no increased risk with maintaining blood pressures in the normal range. The findings cannot be generalized to acute, unstable patients, but the rest the guidelines for blood pressure control should be followed.

Elevated LDL also conferred a high risk of subsequent events in the WASID study [73]. Although the difference failed to reach statistical significance, there were fewer vascular events in patients with LDL $<70$ compared to those with levels $\geq 70$. Based on this data and the SPARCL (stroke prevention by aggressive reduction in cholesterol levels) trial [75], the recommendation is for aggressive lipid lowering with statin in patients with atherosclerotic ischemic stroke.

Another WASID substudy [76] has demonstrated that metabolic syndrome is associated with intracranial atherosclerosis and confers a higher risk of major vascular events in these patients. It is, therefore, an important additional target for primary as well as secondary prevention of intracranial atherosclerotic strokes.

In short, better control of risk factors that promote atherosclerosis like diabetes, tobacco use, and particularly hypertension, dyslipidemia, and metabolic syndrome is warranted in patients with intracranial atherosclerosis.

3.2. Endovascular Therapies. In earlier reports, intracranial angioplasty had a high risk of complications, and the procedure was abandoned [77]. Since then, several factors have led to renewed attention in intracranial angioplasty and stenting. These include advances in microcatheter and balloon technology, high risk of recurrent stroke in patients with intracranial stenosis despite medical management in WASID, and success of endovascular treatments for coronary artery disease [78].

3.3. Angioplasty. Data on angioplasty without stenting is restricted to retrospective studies only. These studies report a high technical success rate (with reduction of stenosis to less than 50\%). However, restenosis rates of up to $50 \%$ have also been reported [79]. This study reports a periprocedural death and stroke rate of $8.3 \%$ and an annual stroke risk of $4.4 \%$. The procedure is associated with complications like intimal dissection, thrombus formation, and vessel rupture as well [80]. Long-term outcome after balloon angioplasty is also yet to be prospectively studied.

3.4. Stenting. Stenting can be used as adjunct to balloon angioplasty to prevent plaque recoiling and to cover a plaque or an intimal flap. It may also reduce the chances of vessel dissection. 
The first prospective trial on stenting was SSYLVIA (stenting of symptomatic atherosclerotic lesions in the vertebral or intracranial arteries) [81] performed in 2004. It was a multicenter trial of use of bare metal stent for symptomatic ICAD. It reported a technical success rate of 95\%, 30-day stroke risk of $6.6 \%$, and a 1 -year stroke risk of $8.5 \%$. There were no deaths reported. Although a restenosis rate of $35 \%$ was seen, most of these were asymptomatic.

The next big trial on stenting used Wingspan [82] which is a flexible, self-expanding, microcatheter deployed stent. This study was also a prospective multicenter trial on 45 patients refractory to medical therapy. Authors reported a technical success rate of $97.7 \%$, a 30 -day stroke or death rate of $4.5 \%$, and a 1 -year stroke rate of $9.3 \%$. Their restenosis rates were better at $7.5 \%$ at 6 months and all were asymptomatic. Long-term follow-up data is also now available for Wingspan [83], and they report restenosis rates in the range of $25 \%-32 \%$ although most is still asymptomatic.

Smaller case series report use of bare metal balloonmounted stents and drug eluting stents. The former are limited by their rigidity and the latter by nonavailability of longterm safety data. Qureshi et al. [84] and Gupta et al. [85] report use of drug coated stents in small number of patients with good short term results in terms of restenosis. These patients need to be on long-term antiplatelet therapy for at least 12 months due to risk of subacute and late thrombosis. Therefore, although technically safe and feasible, there is a need for a long-term study.

A comparative study [86] of primary angioplasty and stenting reveals no difference in terms of stroke free survival at 2 years. Another systematic review [87], however, reports a greater 1-year rate of stroke and death in the angioplasty treated group compared to stent treated patients (20 versus $14 \%)$.

Comparison has also been done between aggressive medical management and endovascular treatment with Wingspan by Jarvis et al. [88]. They report a $13 \%$ rate of stroke or death at 6 months with Wingspan and $16 \%$ with medical management.

Based on this evidence, a prospective, randomized study, stenting versus aggressive medical management for preventing recurrent stroke in intracranial stenosis (SAMMPRIS) [89] was started in November 2008 to compare intensive medical therapy alone with stenting plus intensive medical therapy. The trial has very recently been halted with only $59 \%$ of the planned number of patients recruited. The major reason for this early termination is an unacceptably high complication rate in the stenting arm (14\% patients had a stroke or died in the 30-day period after stenting compared to only $5.8 \%$ in the medical arm) [90]. One important finding coming out from this trial is a much lower stroke recurrence rate than that previously reported. Interestingly, these patients were on combination antiplatelet therapy. Hence, aggressive medical management may well be the way forward for most patients.

3.5. Surgical Treatment. Similar to coronary artery bypass grafting, it is being speculated that a subgroup of patients with intracranial stenosis may benefit from External to
Internal carotid bypass. These patients are the ones who have poor hemodynamic reserve. For assessment of hemodynamic, several modalities are coming up including PET, SPECT, TCD, CT, and MR perfusion. With better use of these modalities, it might be easier to identify patients who may benefit from such a bypass procedure. Two trials are currently underway to determine the effectiveness of such a bypass surgery, one in the US (carotid occlusion surgery studyCOSS) and the other in Japan (Japanese EC-IC bypass trialJET) $[91,92]$. Before the final verdict is out, the procedure cannot be widely recommended (Table 3 ).

\section{Summary of Therapeutic Options}

At present, medical management with antiplatelets is the mainstay of therapy for symptomatic intracranial stenosis. Aspirin is the best studied, but in the acute phase, double antiplatelet agents aspirin/extended release dipyridamole or aspirin plus clopidogrel may be used. For long-term use, combination of Aspirin and clopidogrel may not be as safe and clopidogrel alone should be used. Cilostazol due to its pleiotropic effects has shown promise in Asians.

Aggressive risk factor control is recommended both for symptomatic and asymptomatic disease. This includes aggressive control of blood pressures and LDL cholesterol in particular and of other atherogenic risk factors like diabetes and tobacco use in general.

Endovascular management with angioplasty plus stenting is emerging as a promising modality for high-grade stenosis in patients with failure of medical management. SAMMPRIS has been halted due to adverse effects in the intervention arm [90]. Long-term outcomes of the procedure are yet unknown and restenosis is common, and hence, it cannot be widely recommended.

Surgical treatment with EC-IC bypass is also being evaluated in two randomized trials. Pending results, this modality also has limited application.

\section{Conclusion}

5.1. Known Facts about ICAD. Intracranial atherosclerotic stenosis may be the most common cause of stroke worldwide. Asians are predominantly affected, relative to other races. Various genetic and environmental factors have been implicated as predisposing factors. Although intra-arterial angiography is the gold standard to identify extent of stenosis, noninvasive techniques including TCD ultrasound and TOF MRA have been established as reliable modalities to exclude moderate-to-severe (50\%-99\%) stenosis. Also, CTA and PFI can be used to correctly identify degree of severe stenosis.

In terms of secondary prevention of stroke due to intracranial atherosclerotic stenosis, aspirin continues to be the preferred antiplatelet agent due to its effectiveness comparative to newer antiplatelet therapies. However, among Asians, cilostazol has shown a favorable effect on symptomatic stenosis and is of benefit in terms of fewer bleeds. Combination therapy has shown promise. Moreover, aggressive risk factor management, that is, lowering blood pressure and LDL levels lowers risk of vascular events. Endovascular therapy is still 


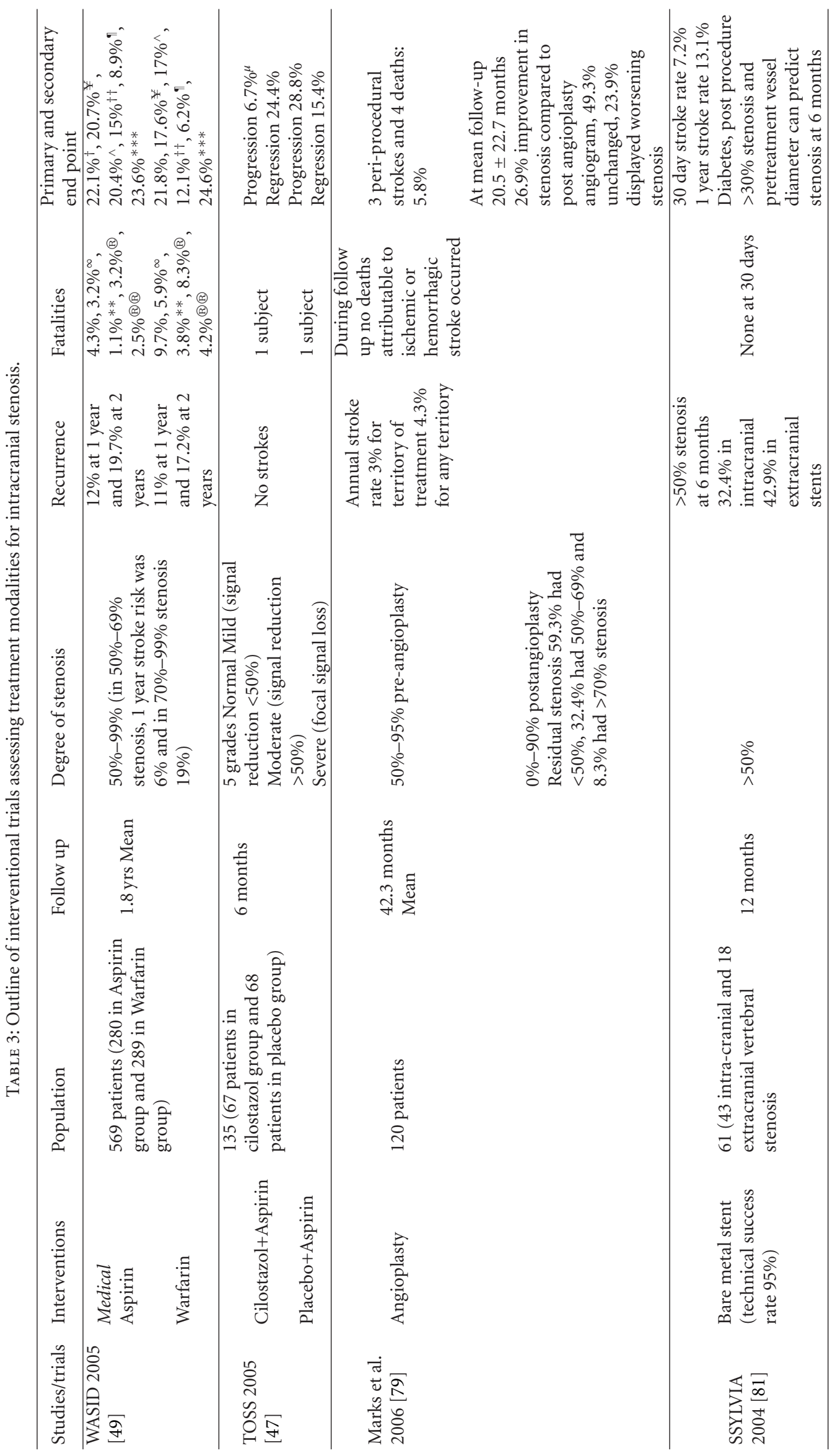




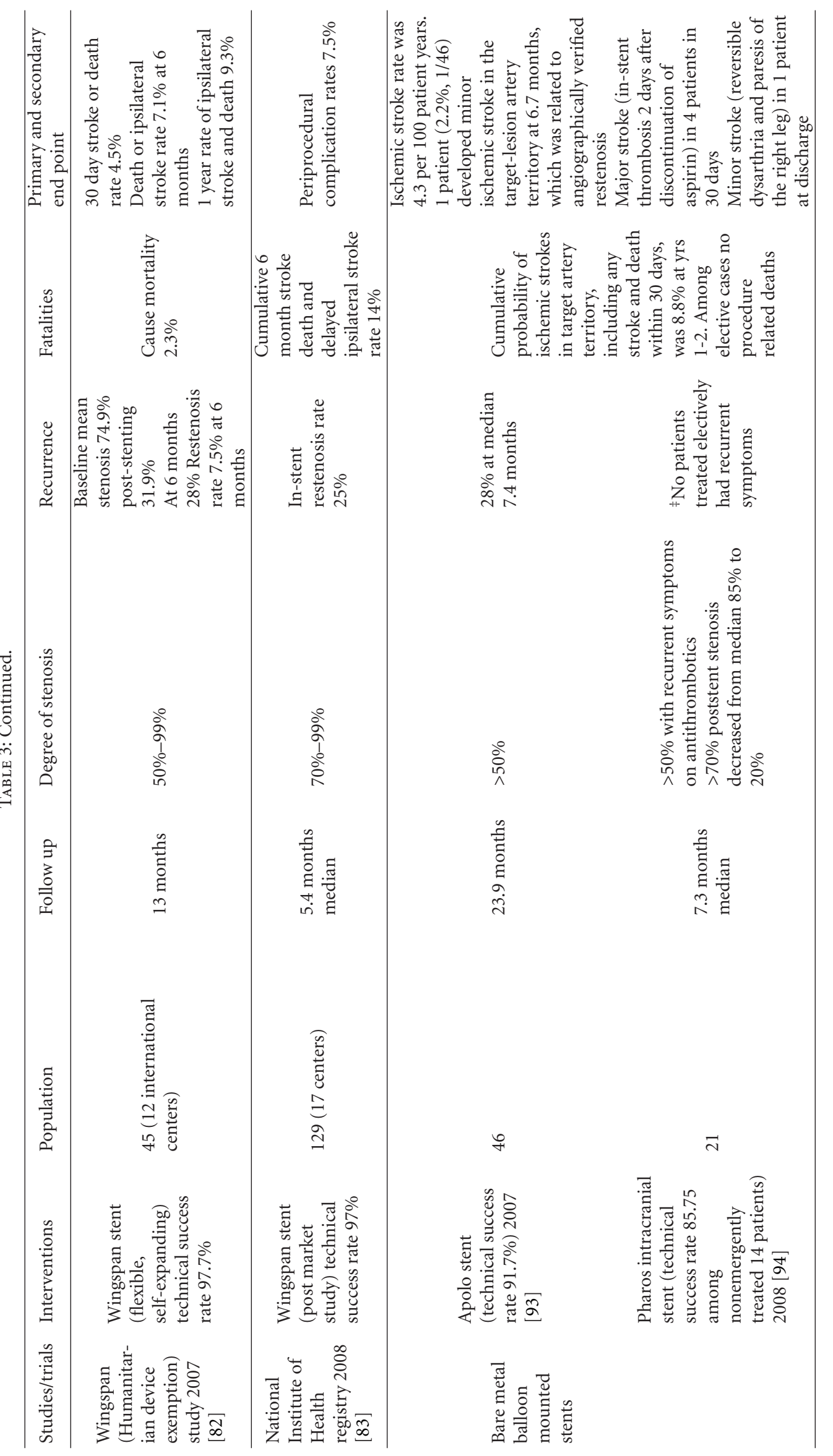




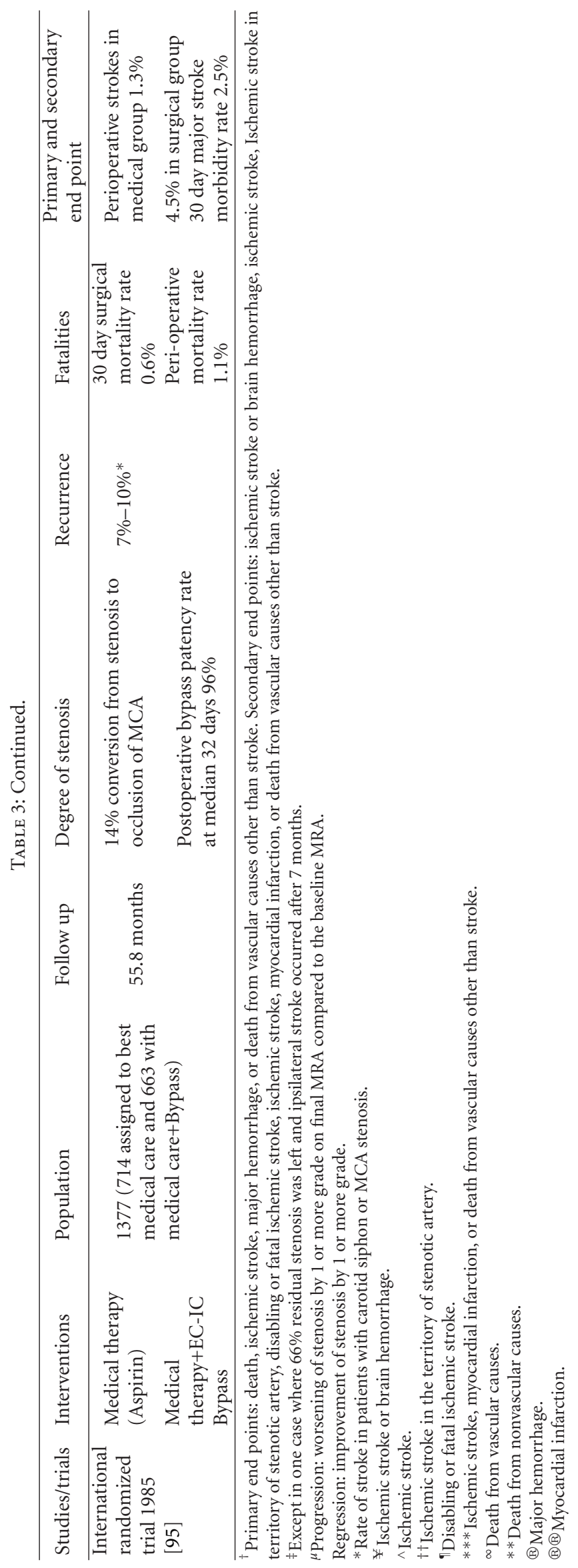


investigational. Surgical treatment, that is, EC-IC bypass, has failed to show any benefit but may be helpful in selected patients with poor hemodynamic reserve.

5.2. Unknown Facts about ICAD. Since Asians are at particular risk of stroke secondary to intracranial atherosclerotic stenosis, studies to locate genetic markers responsible for this racial predominance in lesion distribution would be of interest. Implications of early detection and treatment of asymptomatic intracranial stenosis need to be explored in terms of benefit as a stroke prevention modality. Noninvasive diagnostic techniques that can reliably gauge extent of mild and moderate intracranial stenosis have yet to be identified and are needed. The role of antiplatelet agents other than aspirin, in combination or as single therapy can still be further investigated to advance current medical treatment options. Endovascular therapy is still investigational and is still associated with adverse effects.

5.3. Future Directions. Conducted interventional trials on intracranial atherosclerotic stenosis have so far only been carried out among Caucasians. Since the Asian population is known to be preferentially effected, focused trials need to be carried out to establish treatment modalities that are most effective in this population. Additionally, these may focus on prevention, since intervention is expensive and requires technical expertise in low- and middle-income countries where these resources are scant. Screening for intracranial stenosis needs to be further explored. Noninvasive diagnostic modalities that can reliably identify all degrees of stenoses are needed. For symptomatic patients, this will help in management decisions, and for asymptomatic patients, they may help explore the role of preventive therapy.

\section{Acknowledgments}

M. Khan is a neurovascular fellow whose training is currently funded by Award no. D43TW008660 from the Fogarty International Center. The content is solely the responsibility of the authors and does not necessarily represent the official views of the Fogarty International Center or the National Institutes of Health. A. Kamal is the Principal Investigator for the Karachi Intracranial Stenosis Study (KISS) funded by the Higher Education Commission Government of Pakistan.

\section{References}

[1] P. B. Gorelick, K. S. Wong, H. J. Bae, and D. K. Pandey, "Large artery intracranial occlusive disease: a large worldwide burden but a relatively neglected frontier," Stroke, vol. 39, no. 8, pp. 2396-2399, 2008.

[2] L. K. Wong, "Global burden of intracranial atherosclerosis," International Journal of Stroke, vol. 1, no. 3, pp. 158-159, 2006.

[3] R. L. Sacco, D. Kargman, Q. Gu, and M. C. Zamanillo, "Raceethnicity and determinants of intracranial atherosclerotic cerebral infarction: the Northern Manhattan Stroke Study," Stroke, vol. 26, no. 1, pp. 14-20, 1995.

[4] L. R. Caplan, P. B. Gorelick, and D. B. Hier, "Race, sex and occlusive cerebrovascular disease: a review," Stroke, vol. 17, no. 4, pp. 648-655, 1986.
[5] K. Nishimaru, L. C. McHenry Jr., and J. F. Toole, "Cerebral angiographic and clinical differences in carotid system transient ischemic attacks between American Caucasian and Japanese patients," Stroke, vol. 15, no. 1, pp. 56-59, 1984.

[6] E. Feldmann, N. Daneault, E. Kwan et al., "Chinese-white differences in the distribution of occlusive cerebrovascular disease," Neurology, vol. 40, no. 10, pp. 1541-1545, 1990.

[7] P. McGarry, L. A. Solberg, M. A. Guzman, and J. P. Strong, "Cerebral atherosclerosis in New Orleans. Comparisons of lesions by age, sex, and race," Laboratory Investigation, vol. 52, no. 5, pp. 533-539, 1985.

[8] M. T. Garcia-Rondon and Y. Reyes-Iglesias, "Cerebral angiographic findings and risk factor profile in a hispanic male population," Annals of Neurology, vol. 42, p. 440, 1997.

[9] S. Y. Leung, T. H. K. Ng, S. T. Yuen, I. J. Lauder, and F. C. S. Ho, "Pattern of cerebral atherosclerosis in Hong Kong Chinese: severity in intracranial and extracranial vessels," Stroke, vol. 24, no. 6, pp. 779-786, 1993.

[10] S. J. Lee, S. J. Cho, H. S. Moon et al., "Combined extracranial and intracranial atherosclerosis in Korean patients," Archives of Neurology, vol. 60, no. 11, pp. 1561-1564, 2003.

[11] M. Moussouttas, L. Aguilar, K. Fuentes et al., "Cerebrovascular disease among patients from the Indian subcontinent," Neurology, vol. 67, no. 5, pp. 894-896, 2006.

[12] D. A. de Silva, F.-P. Woon, M.-P. Lee, C. P. L. H. Chen, H.-M. Chang, and M.-C. Wong, "South Asian patients with ischemic stroke: intracranial large arteries are the predominant site of disease," Stroke, vol. 38, no. 9, pp. 2592-2594, 2007.

[13] R. J. Wityk, D. Lehman, M. Klag, J. Coresh, H. Ahn, and B. Litt, "Race and sex differences in the distribution of cerebral atherosclerosis," Stroke, vol. 27, no. 11, pp. 1974-1980, 1996.

[14] Y. N. Huang, S. Gao, S. W. Li et al., "Vascular lesions in Chinese patients with transient ischemic attacks," Neurology, vol. 48, no. 2, pp. 524-525, 1997.

[15] Y. D. Kim, H. Y. Choi, H. J. Cho et al., "Increasing frequency and burden of cerebral artery atherosclerosis in Korean stroke patients," Yonsei Medical Journal, vol. 51, no. 3, pp. 318-325, 2010.

[16] N. C. Suwanwela and A. Chutinetr, "Risk factors for atherosclerosis of cervicocerebral arteries: intracranial versus extracranial," Neuroepidemiology, vol. 22, no. 1, pp. 37-40, 2003.

[17] S. P. Waddy, G. Cotsonis, M. J. Lynn et al., "Racial differences in vascular risk factors and outcomes of patients with intracranial atherosclerotic arterial stenosis," Stroke, vol. 40, no. 3, pp. 719-725, 2009.

[18] T. Uehara, M. Tabuchi, T. Hayashi, H. Kurogane, and A. Yamadori, "Asymptomatic occlusive lesions of carotid and intracranial arteries in Japanese patients with ischemic heart disease: evaluation by brain magnetic resonance angiography," Stroke, vol. 27, no. 3, pp. 393-397, 1996.

[19] L. A. Solberg and P. A. McGarry, "Cerebral atherosclerosis in Negroes and Caucasians," Atherosclerosis, vol. 16, no. 2, pp. 141-154, 1972.

[20] W. Mak, T. S. Cheng, K. H. Chan, R. T. F. Cheung, and S. L. Ho, "A possible explanation for the racial difference in distribution of large-arterial cerebrovascular disease: ancestral European settlers evolved genetic resistance to atherosclerosis, but confined to the intracranial arteries," Medical Hypotheses, vol. 65, no. 4, pp. 637-648, 2005.

[21] K. Asplund, "Antioxidant vitamins in the prevention of cardiovascular disease: a systematic review," Journal of Internal Medicine, vol. 251, no. 5, pp. 372-392, 2002. 
[22] D. P. Vivekananthan, M. S. Penn, S. K. Sapp et al., "Use of antioxidant vitamins for the prevention of cardiovascular disease: metaanalysis of randomized trials," The Lancet, vol. 361, no. 9374, pp. 2017-2023, 2003.

[23] J. Moossy, "Development of cerebral atherosclerosis in various age groups," Neurology, vol. 9, pp. 569-574, 1959.

[24] A. Elbaz and P. Amarenco, "Genetic susceptibility and ischemic stroke," Current Opinion in Neurology, vol. 12, no. 1, pp. 47-55, 1999.

[25] A. Hassan and H. S. Markus, "Genetics and ischaemic stroke," Brain, vol. 123, pp. 1784-1812, 2000.

[26] F. J. Carr, M. W. McBride, H. V. O. Carswell et al., "Genetic aspects of stroke: human and experimental studies," Journal of Cerebral Blood Flow and Metabolism, vol. 22, no. 7, pp. 767$773,2002$.

[27] S. Kunitz, C. Gross, A. Heyman et al., "The pilot stroke data bank: definition, design, and data," Stroke, vol. 15, no. 4, pp. 740-746, 1984.

[28] I. Holme, S. C. Enger, A. Helgeland et al., "Risk factors and raised atherosclerotic lesions in coronary and cerebral arteries. Statistical analysis from the Oslo study," Arteriosclerosis, vol. 1, no. 4, pp. 250-256, 1981.

[29] P. B. Gorelick, L. R. Caplan, D. B. Hier, S. L. Parker, and D. Patel, "Racial differences in the distribution of anterior circulation occlusive disease," Neurology, vol. 34, no. 1, pp. 5459, 1984.

[30] O. Y. Bang, J. W. Kim, J. H. Lee et al., "Association of the metabolic syndrome with intracranial atherosclerotic stroke," Neurology, vol. 65, no. 2, pp. 296-298, 2005.

[31] F. Rincon, R. L. Sacco, G. Kranwinkel et al., "Incidence and risk factors of intracranial atherosclerotic stroke: the Northern Manhattan Stroke Study," Cerebrovascular Diseases, vol. 28, no. 1, pp. 65-71, 2009.

[32] T. N. Turan, A. A. Makki, S. Tsappidi et al., "Risk factors associated with severity and location of intracranial arterial stenosis," Stroke, vol. 41, no. 8, pp. 1636-1640, 2010.

[33] K. S. Wong, S. Gao, Y. L. Chan et al., "Mechanisms of acute cerebral infarctions in patients with middle cerebral artery stenosis: a diffusion-weighted imaging and microemboli monitoring study," Annals of Neurology, vol. 52, no. 1, pp. 74-81, 2002.

[34] A. E. Baird, K. O. Lövblad, G. Schlaug, R. R. Edelman, and S. Warach, "Multiple acute stroke syndrome: marker of embolic disease?" Neurology, vol. 54, no. 3, pp. 674-678, 2000.

[35] J. Bogousslavsky and F. Regli, "Borderzone infarctions distal to internal carotid artery occlusion: prognostic implications," Annals of Neurology, vol. 20, no. 3, pp. 346-350, 1986.

[36] M. del Sette, M. Eliasziw, J. Y. Streifler, V. C. Hachinski, A. J. Fox, and H. J. Barnett, "Internal borderzone infarction: a marker for severe stenosis in patients with symptomatic internal carotid artery disease," Stroke, vol. 31, no. 3, pp. 631-636, 2000.

[37] L. R. Caplan, "Intracranial branch atheromatous disease: a neglected, understudied, and underused concept," Neurology, vol. 39, no. 9, pp. 1246-1250, 1989.

[38] H. P. Adams, H. C. Damasio, S. F. Putman, and A. R. Damasio, "Middle cerebral artery occlusion as a cause of isolated subcortical infarction," Stroke, vol. 14, no. 6, pp. 948-952, 1983.

[39] C. M. Fisher, "Capsular infarcts. the underlying vascular lesions," Archives of Neurology, vol. 36, no. 2, pp. 65-73, 1979.

[40] D. K. Lee, J. S. Kim, S. U. Kwon, S.-H. Yoo, and D.-W. Kang, "Lesion patterns and stroke mechanism in atherosclerotic middle cerebral artery disease: early diffusion-weighted imaging study," Stroke, vol. 36, no. 12, pp. 2583-2588, 2005.
[41] L. C. Turtzo, R. F. Gottesman, and R. H. Llinas, "Diffusionweighted imaging showing "Pearls" predicts large-vessel disease as stroke etiology," Cerebrovascular Diseases, vol. 28, no. 1, pp. 49-54, 2009.

[42] H. Yamauchi, R. Nish, T. Higashi, S. Kagawa, and H. Fukuyama, "Hemodynamic compromise as a cause of internal border-zone infarction and cortical neuronal damage in atherosclerotic middle cerebral artery disease," Stroke, vol. 40, no. 12, pp. 3730-3735, 2009.

[43] C. P. Derdeyn, W. J. Powers, and R. L. Grubb Jr., "Hemodynamic effects of middle cerebral artery stenosis and occlusion," American Journal of Neuroradiology, vol. 19, no. 8, pp. 14631469, 1998.

[44] D. W. Kang, J. A. Chalela, M. A. Ezzeddine, and S. Warach, "Association of ischemic lesion patterns on early diffusionweighted imaging with TOAST stroke subtypes," Archives of Neurology, vol. 60, no. 12, pp. 1730-1734, 2003.

[45] J. Bogousslavsky, "Double infarction in one cerebral hemisphere," Annals of Neurology, vol. 30, no. 1, pp. 12-18, 1991.

[46] P. T. Akins, T. K. Pilgram, D. T. Cross III, and C. J. Moran, "Natural history of stenosis from intracranial atherosclerosis by serial angiography," Stroke, vol. 29, no. 2, pp. 433-438, 1998.

[47] S. U. Kwon, Y. J. Cho, J. S. Koo et al., "Cilostazol prevents the progression of the symptomatic intracranial arterial stenosis: the multicenter double-blind placebo-controlled trial of cilostazol symptomatic intracranial arterial stenosis," Stroke, vol. 36, no. 4, pp. 782-786, 2005.

[48] S. U. Kwon, D. W. Kang, J. M. Park et al., "Trial of efficacy and safety of cilostazol on the progression of symptomatic intracranial stenosis comparing clopidogrel: trial of cilostazol in symptomatic intracranial stenosis-2 (TOSS-2)," Cerebrovascular Diseases, vol. 27, supplement 6, pp. 10-11, 2009.

[49] M. I. Chimowitz, M. J. Lynn, H. Howlett-Smith et al., "Comparison of warfarin and aspirin for symptomatic intracranial arterial stenosis," The New England Journal of Medicine, vol. 352, no. 13, pp. 1305-1316, 2005.

[50] S. E. Kasner, M. I. Chimowitz, M. J. Lynn et al., "Predictors of ischemic stroke in the territory of a symptomatic intracranial arterial stenosis," Circulation, vol. 113, no. 4, pp. 555-563, 2006.

[51] M. Mazighi, R. Tanasescu, X. Ducrocq et al., "Prospective study of symptomatic atherothrombotic intracranial stenoses: the GESICA study," Neurology, vol. 66, no. 8, pp. 1187-1191, 2006.

[52] D. S. Liebeskind, G. A. Cotsonis, J. L. Saver et al., "Collateral dramatically alter stroke risk in intracranial atherosclerosis," Annals of Neurology, vol. 69, no. 6, pp. 963-974, 2011.

[53] J. T. Fifi, P. M. Meyers, S. D. Lavine et al., "Complications of modern diagnostic cerebral angiography in an academic medical center," Journal of Vascular and Interventional Radiology, vol. 20, no. 4, pp. 442-447, 2009.

[54] S. M. Debrey, H. Yu, J. K. Lynch et al., "Diagnostic accuracy of magnetic resonance angiography for internal carotid artery disease: a systematic review and meta-analysis," Stroke, vol. 39, no. 8, pp. 2237-2248, 2008.

[55] J. E. Heiserman, B. P. Drayer, P. J. Keller et al., "Intracranial vascular stenosis and occlusion: evaluation with three-dimensional time-of-flight MR angiography," Radiology, vol. 185, no. 3, pp. 667-673, 1992.

[56] Y. Korogi, M. Takahashi, N. Mabuchi et al., "Intracranial vascular stenosis and occlusion: diagnostic accuracy of threedimensional, fourier transform, time-of-flight MR angiography," Radiology, vol. 193, no. 1, pp. 187-193, 1994. 
[57] C. G. Choi, D. H. Lee, J. H. Lee et al., "Detection of intracranial atherosclerotic steno-occlusive disease with 3D time-of-flight magnetic resonance angiography with sensitivity encoding at 3T," American Journal of Neuroradiology, vol. 28, no. 3, pp. 439-446, 2007.

[58] J. F. Arenillas, "Intracranial atherosclerosis: current concepts," Stroke, vol. 42, supplement 1, pp. S20-S23, 2011.

[59] S. Bash, J. P. Villablanca, R. Jahan et al., "Intracranial vascular stenosis and occlusive disease: evaluation with CT angiography, MR angiography, and digital subtraction angiography," American Journal of Neuroradiology, vol. 26, no. 5, pp. 10121021, 2005.

[60] E. Feldmann, J. L. Wilterdink, A. Kosinski et al., “The stroke outcomes and neuroimaging of intracranial atherosclerosis (SONIA) trial," Neurology, vol. 68, no. 24, pp. 2099-2106, 2007.

[61] G. Fürst, A. Saleh, F. Wenserski et al., "Reliability and validity of noninvasive imaging of internal carotid artery pseudoocclusion," Stroke, vol. 30, no. 7, pp. 1444-1449, 1999.

[62] A. M. Demchuk, I. Christou, T. H. Wein et al., "Accuracy and criteria for localizing arterial occlusion with transcranial doppler," Journal of Neuroimaging, vol. 10, no. 1, pp. 1-12, 2000.

[63] International Stroke Trial Collaborative Group, “The International Stroke Trial (IST): a randomised trial of aspirin, subcutaneous heparin, both, or neither among 19435 patients with acute ischaemic stroke," The Lancet, vol. 349, no. 9065, pp. 1569-1581, 1997.

[64] Z.-M. Chen, "CAST: randomised placebo-controlled trial of early aspirin use in 20,000 patients with acute ischaemic stroke," The Lancet, vol. 349, no. 9066, pp. 1641-1649, 1997.

[65] S. E. Kasner, M. J. Lynn, M. I. Chimowitz et al., "Warfarin aspirin symptomatic intracranial disease (WASID) trial investigators. Warfarin vs aspirin for symptomatic intracranial stenosis: subgroup analyses from WASID," Neurology, vol. 67, no. 7, pp. 1275-1278, 2006.

[66] H. C. Diener, L. Cunha, C. Forbes, J. Sivenius, P. Smets, and A. Lowenthal, "European stroke prevention study 2. Dipyridamole and acetylsalicylic acid in the secondary prevention of stroke," Journal of the Neurological Sciences, vol. 143, no. 1-2, pp. 1-13, 1996.

[67] CAPRIE Steering Committee, "A randomised, blinded, trial of clopidogrel versus aspirin in patients at risk of ischaemic events (CAPRIE)," The Lancet, vol. 348, no. 9038, pp. 13291339, 1996.

[68] R. L. Sacco, H. C. Diener, S. Yusuf et al., "Aspirin and extended-release dipyridamole versus clopidogrel for recurrent stroke," The New England Journal of Medicine, vol. 359, no. 12, pp. 1238-1251, 2008.

[69] R. J. Adams, G. Albers, M. J. Alberts et al., "Update to the AHA/ASA recommendations for the prevention of stroke in patients with stroke and transient ischemic attack," Stroke, vol. 39, no. 5, pp. 1647-1652, 2008.

[70] K. Wong, C. Chen, J. Fu et al., "Clopidogrel plus aspirin versus aspirin alone for reducing embolisation in patients with acute symptomatic cerebral or carotid artery stenosis (CLAIR study): a randomised, open-label, blinded-endpoint trial," The Lancet Neurology, vol. 9, no. 5, pp. 489-497, 2010.

[71] Y. Huang, Y. Cheng, J. Wu et al., "Cilostazol as an alternative to aspirin after ischaemic stroke: a randomised, double-blind, pilot study," The Lancet Neurology, vol. 7, no. 6, pp. 494-499, 2008.
[72] Y. Shinohara, Y. Katayama, S. Uchiyama et al., "Cilostazol for prevention of secondary stroke (CSPS 2): an aspirincontrolled, double-blind, randomised non-inferiority trial," The Lancet Neurology, vol. 9, no. 10, pp. 959-968, 2010.

[73] S. Chaturvedi, T. N. Turan, M. J. Lynn et al., "Risk factor status and vascular events in patients with symptomatic intracranial stenosis," Neurology, vol. 69, no. 22, pp. 2063-2068, 2007.

[74] T. N. Turan, G. Cotsonis, M. J. Lynn, S. Chaturvedi, and M. Chimowitz, "Relationship between blood pressure and stroke recurrence in patients with intracranial arterial stenosis," Circulation, vol. 115, no. 23, pp. 2969-2975, 2007.

[75] P. Amarenco, J. Bogousslavsky, A. Callahan III et al., "Highdose atorvastatin after stroke or transient ischemic attack," The New England Journal of Medicine, vol. 355, no. 6, pp. 549-559, 2006.

[76] B. Ovbiagele, J. L. Saver, M. J. Lynn, and M. Chimowitz, "Impact of metabolic syndrome on prognosis of symptomatic intracranial atherostenosis," Neurology, vol. 66, no. 9, pp. 1344-1349, 2006.

[77] T. M. Sundt Jr., H. C. Smith, J. K. Campbell et al., "Transluminal angioplasty for basilar artery stenosis," Mayo Clinic Proceedings, vol. 55, no. 11, pp. 673-680, 1980.

[78] T. N. Turan, C. P. Derdeyn, D. Fiorella, and M. I. Chimowitz, "Treatment of atherosclerotic intracranial arterial stenosis," Stroke, vol. 40, no. 6, pp. 2257-2261, 2009.

[79] M. P. Marks, J. C. Wojak, F. Al-Ali et al., "Angioplasty for symptomatic intracranial stenosis: clinical outcome," Stroke, vol. 37, no. 4, pp. 1016-1020, 2006.

[80] M. Hartmann and O. Jansen, "Angioplasty and stenting of intracranial stenosis," Current Opinion in Neurology, vol. 18, no. 1, pp. 39-45, 2005.

[81] SSYLVIA Study Investigators, "Stenting of symptomatic atherosclerotic lesions in the vertebral or intracranial arteries (SSYLVIA): study results," Stroke, vol. 35, no. 6, pp. 1388-1392, 2004.

[82] A. Bose, M. Hartmann, H. Henkes et al., "A novel, self-expanding, nitinol stent in medically refractory intracranial atherosclerotic stenoses: the wingspan study," Stroke, vol. 38, no. 5, pp. 1531-1537, 2007.

[83] O. O. Zaidat, R. Klucznik, M. J. Alexander et al., “The NIH registry on use of the wingspan stent for symptomatic 70-99\% intracranial arterial stenosis," Neurology, vol. 70, no. 17, pp. 1518-1524, 2008.

[84] A. I. Qureshi, J. F. Kirmani, H. M. Hussein et al., "Early and intermediate-term outcomes with drug-eluting stents in highrisk patients with symptomatic intracranial stenosis," Neurosurgery, vol. 59, no. 5, pp. 1044-1051, 2006.

[85] R. Gupta, F. Al-Ali, A. J. Thomas et al., "Safety, feasibility, and short-term follow-up of drug-eluting stent placement in the intracranial and extracranial circulation," Stroke, vol. 37, no. 10, pp. 2562-2566, 2006.

[86] F. Siddiq, G. Vazquez, M. Z. Memon et al., "Comparison of primary angioplasty with stent placement for treating symptomatic intracranial atherosclerotic diseases: a multicenter study," Stroke, vol. 39, no. 9, pp. 2505-2510, 2008.

[87] F. Siddiq, M. Z. Memon, G. Vazquez, A. Safdar, and A. I. Qureshi, "Comparison between primary angioplasty and stent placement for symptomatic intracranial atherosclerotic disease: meta-analysis of case series," Neurosurgery, vol. 65, no. 6, pp. 1024-1033, 2009.

[88] A. L. Jarvis, M. Chimowitz, M. J. Lynn et al., "Outcome of patients with $50-99 \%$ intracranial stenosis and TIA or stroke on antithrombotic therapy treated medically vs. stenting," in Proceedings of the 60th Annual Meeting of the American Academy of Neurology, Chicago, Ill, USA, April 2008. 
[89] C. P. Derdeyn and M. I. Chimowitz, "Angioplasty and stenting for atherosclerotic intracranial stenosis: rationale for a randomized clinical trial," Neuroimaging Clinics of North America, vol. 17, no. 3, pp. 355-363, 2007.

[90] K. Dmyterko Jr., "SAMMPRIS trial halted after high risk of stroke/death found," Industry News, April 2011.

[91] R. L. Grubb Jr., W. J. Powers, C. P. Derdeyn, H. P. Adams Jr., and W. R. Clarke, "The carotid occlusion surgery study," Neurosurgical Focus, vol. 14, no. 3, p. e9, 2003.

[92] S. Mizumura, J. Nakagawara, M. Takahashi et al., "Threedimensional display in staging hemodynamic brain ischemia for JET study: objective evaluation using SEE analysis and 3DSSP display," Annals of Nuclear Medicine, vol. 18, no. 1, pp. 13-21, 2004.

[93] W.-J. Jiang, X.-T. Xu, M. Jin, B. Du, K.-H. Dong, and J.-P. Dai, "Apollo stent for symptomatic atherosclerotic intracranial stenosis: study results," American Journal of Neuroradiology, vol. 28, no. 5, pp. 830-834, 2007.

[94] W. Kurre, J. Berkefeld, M. Sitzer, T. Neumann-Haefelin, and R. du Mesnil de Rochemont, "Treatment of symptomatic high-grade intracranial stenoses with the balloon-expandable Pharos stent: initial experience," Neuroradiology, vol. 50, no. 8, pp. 701-708, 2008.

[95] The EC/IC Bypass Study Group, "Failure of extracranial-intracranial arterial bypass to reduce the risk of ischemic Stroke. Results of an international randomized trial," The New England Journal of Medicine, vol. 313, no. 19, pp. 1191-1200, 1985. 


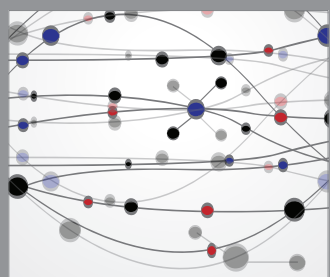

The Scientific World Journal
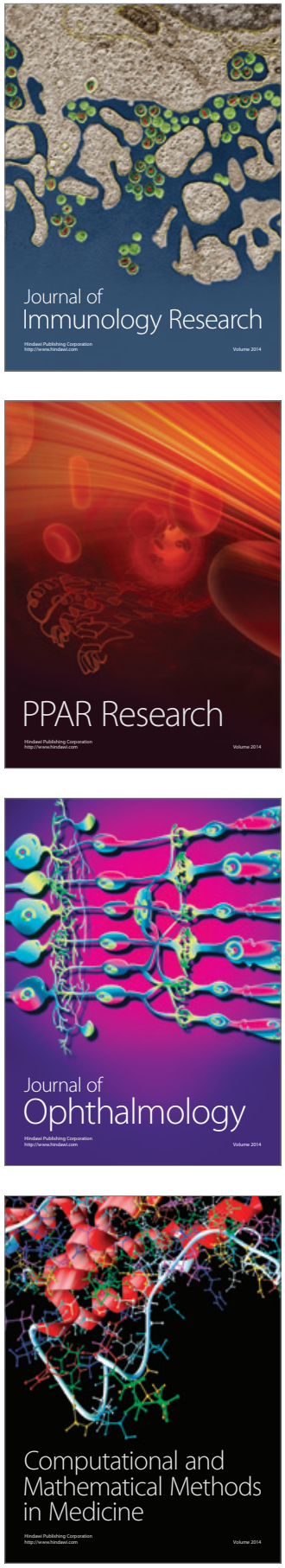

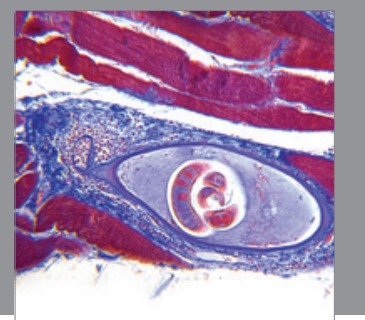

Gastroenterology

Research and Practice
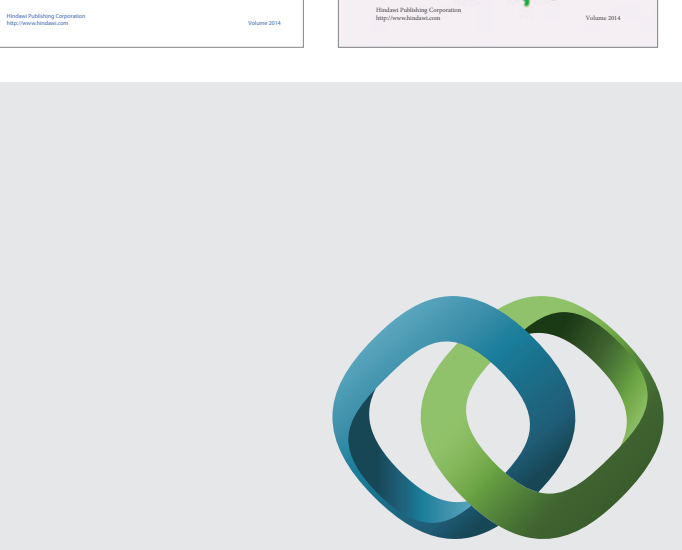

\section{Hindawi}

Submit your manuscripts at

http://www.hindawi.com
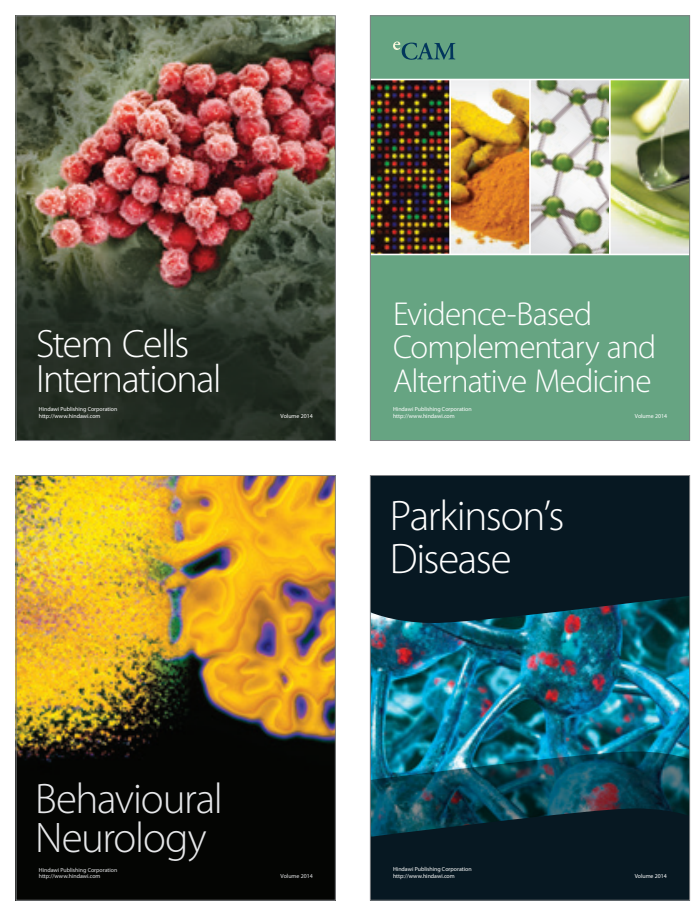

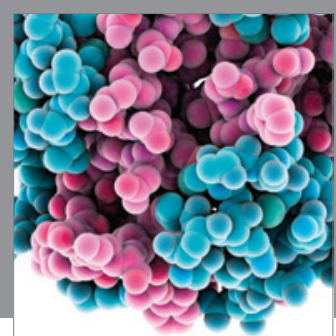

Journal of
Diabetes Research

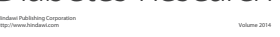

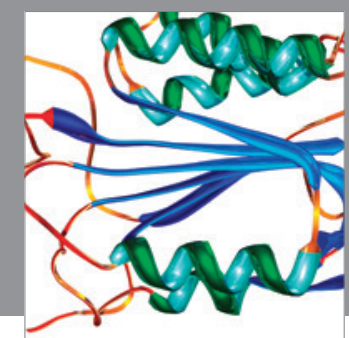

Disease Markers
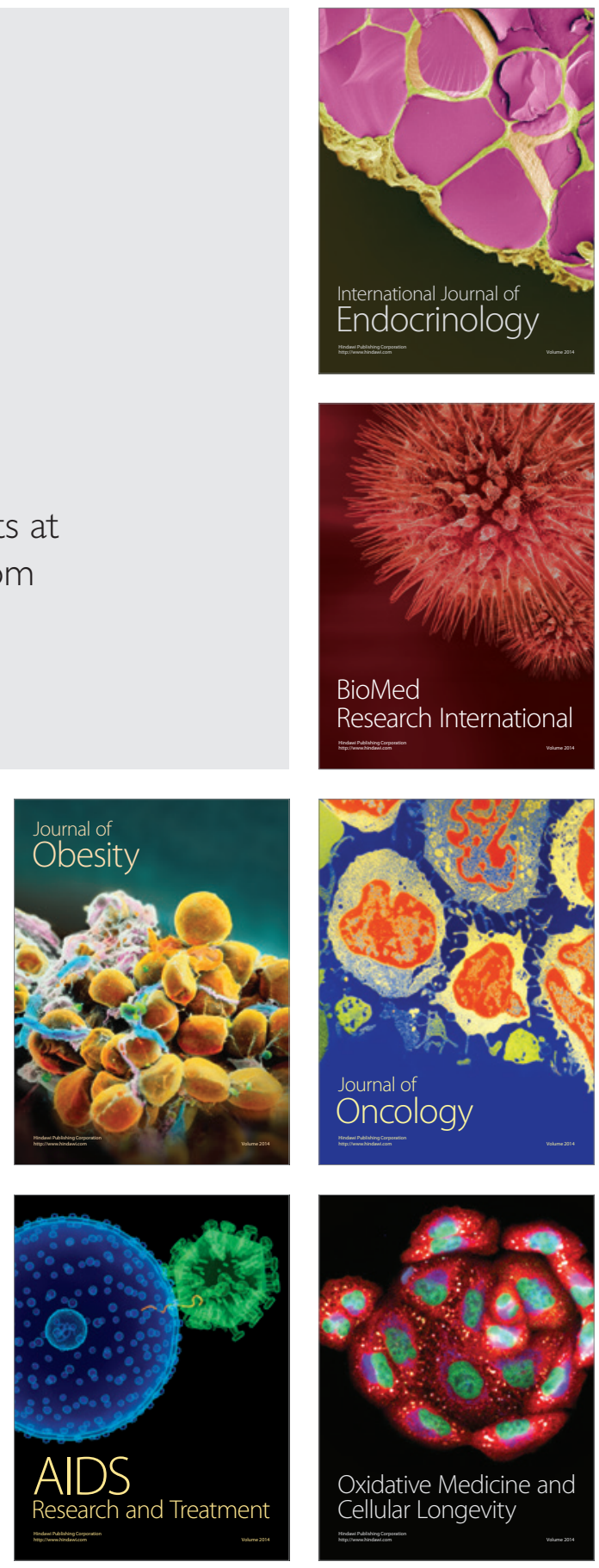